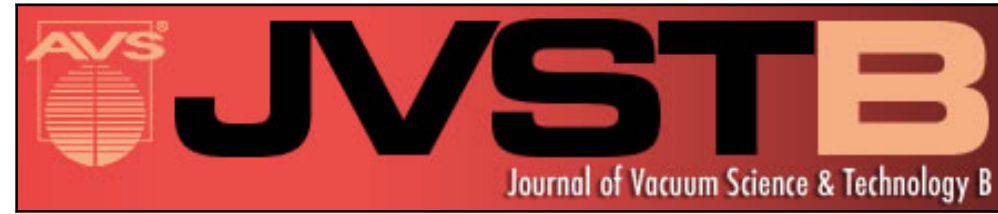

\title{
Rigid organic molds for nanoimprint lithography by replica molding of high glass transition temperature polymers
}

Dario Pisignano, Stefania D’Amone, Giuseppe Gigli, and Roberto Cingolani

Citation: Journal of Vacuum Science \& Technology B 22, 1759 (2004); doi: 10.1116/1.1767108

View online: http://dx.doi.org/10.1116/1.1767108

View Table of Contents: http://scitation.aip.org/content/avs/journal/jvstb/22/4?ver=pdfcov

Published by the AVS: Science \& Technology of Materials, Interfaces, and Processing

\section{Articles you may be interested in}

High-density pattern transfer via roll-to-roll ultraviolet nanoimprint lithography using replica mold

J. Vac. Sci. Technol. B 30, 06FB07 (2012); 10.1116/1.4758922

Double-anchoring fluorinated molecules for antiadhesion mold treatment in UV nanoimprint lithography

J. Vac. Sci. Technol. B 27, 2873 (2009); 10.1116/1.3245993

Ultraviolet nanoimprinted polymer nanostructure for organic light emitting diode application

Appl. Phys. Lett. 92, 223307 (2008); 10.1063/1.2939554

Polymer time constants during low temperature nanoimprint lithography

J. Vac. Sci. Technol. B 23, 2963 (2005); 10.1116/1.2121727

Defect analysis in thermal nanoimprint lithography

J. Vac. Sci. Technol. B 21, 2765 (2003); 10.1116/1.1629289

\section{Instruments for Advanced Science}

Contact Hiden Analytical for further details: w www.HidenAnalytical.com E info@hiden.co.uk CLICK TO VIEW our product catalogue

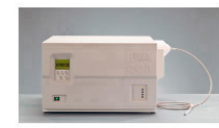

Gas Analysis

dynamic measurement of reaction gas streams catalysis and thermal analysis

molecular beam studies

fermentation, enuironmental and ecological studies

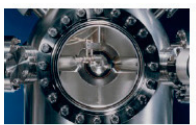

Surface Science UHVTPD SIMS

end point detection in ion beam etch elemental imaging - surface mapping

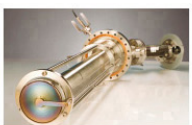

Plasma Diagnostics , plasma source characterization etch and deposition process reaction kinetic studies , analysis of neutral and radical species

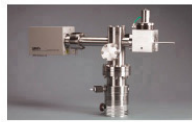

Vacuum Analysis - partial pressure measurement and contro of process gases reactive sputter process control vacuum diagnostics vacuum coating process monitoring 


\title{
Rigid organic molds for nanoimprint lithography by replica molding of high glass transition temperature polymers
}

\author{
Dario Pisignano, ${ }^{\text {a) }}$ Stefania D’Amone, Giuseppe Gigli, and Roberto Cingolani \\ NNL, National Nanotechnology Laboratory of Istituto Nazionale di Fisica della Materia (INFM) \\ c/o Dipartimento di Ingegneria dell'Innovazione, Università di Lecce, via Arnesano, I-73100 Lecce, Italy
}

(Received 19 December 2003; accepted 5 May 2004; published 14 July 2004)

\begin{abstract}
The glass transition of polycarbonate resins of high softness temperature is exploited to realize rigid polymeric replicas of master patterns, with no need for antisticking layers and applied pressure. Such replicas enable the transfer of patterns onto polymers having a lower glass-transition temperature by nanoimprint lithography. As a demonstration, we show the pattern transfer onto poly(methylmethacrylate), which demonstrates good fidelity and remarkable simplicity of the process. (C) 2004 American Vacuum Society. [DOI: 10.1116/1.1767108]
\end{abstract}

\section{INTRODUCTION}

Nanoimprint lithography (NIL) (Refs. 1 and 2) exploits the glass transition of organic compounds to transfer, with very high fidelity, master patterns realized by photo- or electron-beam lithography. As opposed to soft lithographytype methods, such as microcontact printing ${ }^{3}$ or capillary force lithography, ${ }^{3-5}$ which are based on the conformal contact between the target polymer and the elastomeric replica of the original master, NIL employs rigid molds under pressure. After placing the mold onto the polymeric film, the system is driven above the glass transition temperature, $T_{g}$, of the target and a pressure in the range of $10^{2}-10^{4} \mathrm{psi}$ is applied. In this way, the polymer takes the shape of the master. Different physical mechanisms are involved in such a process, including the elastic response of the polymer, its viscous response to external stress, and its anelastic behavior determined by the flexibility of the macromolecules. ${ }^{6} \mathrm{~A}$ dominant role is played by the viscous flow of the polymeric molecules under pressure, due to the high mobility, i.e., the low viscosity, occurring above $T_{g}$. The final and most critical step is the subsequent cooling down below $T_{g}$, as the vitrification which occurs upon cooling freezes the pattern into the target polymer, so that the mold can be finally released.

The polymeric pattern, achieved without exposure to radiation, development, or etching processes, can then be exploited as a mask for etching or lift-off processes, or as a device itself, as in the case of functional materials like organic semiconductors. The flexibility, the large-area operation, and the low cost make NIL a very promising route to nanofabrication. The realization by imprinting of direct three-dimensional patterning, ${ }^{7}$ nanoscale field effect transistors, ${ }^{8}$ metal-semiconductor-metal photodetectors, ${ }^{9}$ and sub-10 nm structures ${ }^{10}$ have been demonstrated so far.

The peculiarity of soft lithography ${ }^{11}$ is to replicate the pattern, instead of fabricating it. Nevertheless, to date, the realization of high-resolution molds for NIL has to be carried out by traditional, and expensive, lithographic techniques. The master replication into thermocurable polymers has been

\footnotetext{
a) Also at Dipartimento di Fisica, Università di Lecce, via Arnesano, I-73100 Lecce, Italy; electronic mail: dario.pisignano@unile.it
}

recently suggested. ${ }^{12}$ Such a replica molding process exploits the in situ polymerization of a thermosetting compound, similar to the procedure used to produce elastomeric replicas in poly(dimethylsiloxane). To optimize the pattern transfer to such replicas, ${ }^{12}$ which are rigid and, hence, suitable for NIL, a pressure of about 1500 psi is applied during polymerization in combination with the deposition of a Teflon ${ }^{\circledR}$-like antisticking layer on the master.

Reproducing the master patterns into replicated molds, possibly without using ultraviolet radiation, external pressure, and antisticking layers, is a very important issue for nanoimprinting. This would prolong the life of the master, enable positive-tone imprinting, and lower the overall cost of the lithography. In this article, we suggest a route to realize rigid polymeric replicas to be used as molds for NIL. Our approach is based on a simple replica molding procedure, exploiting the glass transition of a polymer with high $T_{g}$, which is subsequently employed for NIL of other compounds of lower $T_{g}$. So, the same physical mechanism (glass transition of amorphous compounds and subsequent cooling) is exploited in both steps of lithography: Replica molding and imprinting. In the master replication, no pressure and no antisticking layer are needed.

\section{EXPERIMENT}

Master patterns were fabricated onto quartz substrates by electron-beam lithography with a Leica LION LVI system working at an acceleration energy of $2.5 \mathrm{keV}$, followed by a reactive ion etching. They have area of about $2 \mathrm{~cm}^{2}$ and spatial profile of period, $\Lambda \cong 1.2 \mu \mathrm{m}$, with features of about $300 \mathrm{~nm}$ and an etch depth between 200 and $500 \mathrm{~nm}$. Rigid polymeric replicas were realized by placing a commercial polycarbonate resin (Lexan 123R, GE Plastics Europe, The Netherlands, melting point between 280 and $300^{\circ} \mathrm{C}$ ) onto the masters on a hot plate set at $300^{\circ} \mathrm{C}$. The effective temperature on the upper surface of the master was about $210^{\circ} \mathrm{C}$, and the time for a complete pattern transfer was about $5 \mathrm{~min}$. After the system cools down, the rigid polymeric replica was easily separated from the original master by hand. A small downward force (around $0.5 \mathrm{kN}$ for a $1 \mathrm{~mm}^{2}$ large replica) can be applied in order to obtain polymer elements with a flatter upper side; however, the pattern transfer to polycar- 
bonate is successful also without external pressure. No tendency of the polymer to adhere to the master during the replication was observed, which allowed us to not use antisticking layers.

The NIL process was then performed on films of poly(methylmethacrylate) (PMMA) with a molecular weight of $120 \mathrm{~K}$ (Aldrich Chem., MO, $T_{g} \cong 120^{\circ} \mathrm{C}$ ) and $950 \mathrm{~K}$ (Allresist, Germany, $T_{g} \cong 130^{\circ} \mathrm{C}$ ), spin cast on $\mathrm{GaAs}$ and $\mathrm{Si}$ substrates with a thickness between $280 \mathrm{~nm}$ and about $1 \mu \mathrm{m}$. In order to prevent the adhesion of the polymer replica to the target during NIL, we carried out NIL after spin casting on polycarbonate a solution of octadecyltrichlorosilane (OTS) in 2,2,4-trimethylpentane $(4: 1000 \mathrm{v} / \mathrm{v})$ at $6000 \mathrm{rpm}$ for $40 \mathrm{~s}$. In contrast to the elastomeric elements, which spontaneously adhere to the target materials, rigid replicas need an applied pressure to transfer the pattern to the target polymers. The NIL process on PMMA $950 \mathrm{~K}$ and $120 \mathrm{~K}$ was performed at temperatures of 100 and $120^{\circ} \mathrm{C}$, respectively, with a pressure around $10^{4}$ psi applied by a precision manual press (PW100 $\mathrm{P} / \mathrm{O} /$ Weber, Germany). ${ }^{13}$

Scanning electron microscopy (SEM) and atomic force microscopy (AFM) (Digital NanoScope, CA) measurements were carried out on both the replicas and the imprinted samples. A few nm thick metal layer was thermally evaporated onto the polymeric replicas to facilitate the morphological investigation.

\section{RESULTS AND DISCUSSION}

\section{A. Physical mechanism of nanoimprint lithography}

Even if the microscopic origin of the glass transition phenomenology is still an open issue of condensed matter physics, it is well known that, with decreasing temperature, the structural relaxation time, $\tau$, and the viscosity, $\eta$, undergo a steep increase, as a consequence of the drastic decrease of the mobility of the molecules constituting the liquid. In fact, the temperature dependence of the relaxation time is usually well described by the Vogel-Fulcher-Tamman (VFT) law: ${ }^{14,15}$

$$
\tau(T)=\tau_{0} \exp \left[D T_{0} /\left(T-T_{0}\right)\right],
$$

where $\tau_{0}$ is the relaxation time at very high temperatures, $D$ is referred to as the strength parameter, and $T_{0}$ is the temperature at which the divergence of the relaxation time is predicted. By employing a sufficiently high cooling rate, the temperature variation may become faster than the characteristic relaxation time by which the system returns to the thermodynamic equilibrium after an external perturbation. In such a condition, one is able to freeze the disordered structure, which is typical of liquids, into a solidlike matrix.

Besides other factors, ${ }^{6}$ the pattern transfer during the imprinting process arises from a similar mechanism: The time scale of the structural rearrangement of the target polymer ("relaxation") has to be shorter than the interval during which the system is kept at high temperature ("observation" window) and during which the target takes the shape of the mold. Consequently, phenomenological laws, such as the VFT equation, can be employed to determine the tempera-

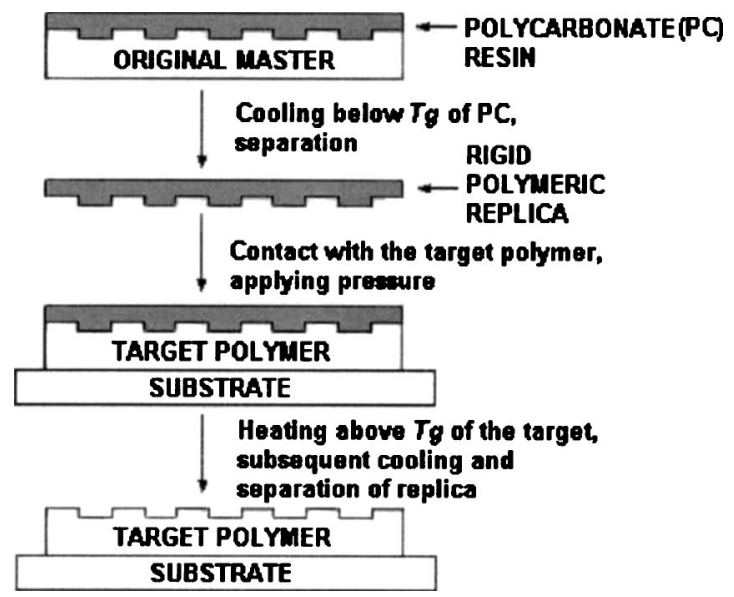

FIG. 1. Schematic diagram of the process of master replication and imprinting (features are not in scale).

ture, $T$, needed for imprinting. For instance, if one wants a satisfactory pattern transfer by an imprinting time of $10 \mathrm{~s}$, one could choose the temperature:

$$
T=T_{0}+\frac{D T_{0}}{\ln \left[100 / \tau_{0}\right]}=T_{0}+\frac{D T_{0}}{F\left(D, T_{0}, T_{g}\right)},
$$

where $\tau_{0}$ is expressed in seconds, and $F(x, y, z) \cong x y / z-y$ -2.3 . Lower temperatures can, of course, be applied with a longer process duration.

In this work, we exploit the glass transition phenomenology not only for the final pattern transfer to the target, but also for the replica molding. This allows a master replication without both applied pressure and antisticking layers. The complete process of master replication and imprinting, summarized in Fig. 1, requires less than $20 \mathrm{~min}$ and is one of the most rapid pattern transfers so far reported for soft lithography.

\section{B. Fabrication of rigid polymeric molds}

A suitable material for the polymeric replicas should have a softness temperature considerably higher than that of all the target polymers to be patterned by NIL. In other words, at the temperature of NIL, the structural relaxation time of the material of the replica has to be much longer than that of the target, in order to ensure the rigidity under the pressure applied to transfer the pattern. To elucidate the working mechanisms of the proposed method, namely the use of an intermediate high- $T_{g}$ polymer mold to transfer the pattern, we chose three prototype systems with physically reliable temperature dynamics parameters (Fig. 2). A detailed list of these structural parameters for a very wide range of compounds can be found in Ref. 15. Here, we consider three molecules whose relaxation times are described by Eq. (1), having $T_{g} \cong 190^{\circ} \mathrm{C}$ [Fig. 2(a)—similar to high- $T_{g}$ polycarbonates], $170^{\circ} \mathrm{C}$ [Fig. 2(b) - like some polystyrenes], and $140^{\circ} \mathrm{C}$ [Fig. 2(c) —analogous to PMMA], respectively. As indicated in Fig. 2, besides the contribution of $\tau_{0}$, the structural dynamics of a polymer [Fig. 2(a)] is much slower than that of polymer [Fig. 2(b)] depending on the value of $D$, and 


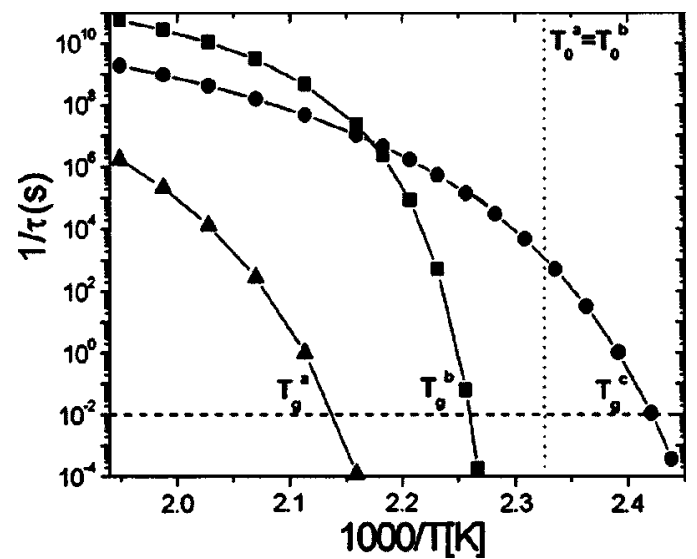

Fig. 2. Temperature dynamics of the structural relaxation times, $\tau$, of three polymers, a high- $T_{g}$ one (a), a medium- $T_{g}$ one (b), and a low- $T_{g}$ one [described according to Eq. (1)]. All three VFT laws have the same $\tau_{0}\left(10^{-13} \mathrm{~s}\right)$ and $\left(D, T_{0}\right)$ parameters as follows: $(3,430 \mathrm{~K})(\mathrm{a}) ;(1,430 \mathrm{~K})(\mathrm{b})$; and $(3$, $380 \mathrm{~K})(\mathrm{c})$. The horizontal dashed line indicated the relaxation time (i.e., $\left.10^{2} \mathrm{~s}\right)$ corresponding to $T_{g}$. The vertical dotted line indicates the $T_{0}$ temperature for polymers (a) and (b). The $T_{0}$ temperature for polymer (c) is out of the plot.

much slower than polymer [Fig. 2(c)] depending on the value of $T_{0}$. These behaviors are very general: The molecular structure (namely, the molecular weight and the degree of steric hindrance) determines how easily the polymer network is frozen upon decreasing temperature, and higher values of $T_{g}$ are associated with a slower structural dynamics.

Consequently, polymers [Fig. 2(a)], characterized by a slower dynamics (higher $T_{g}$ ) can be employed to fabricate molds for NIL processes having as target polymers, Figs. 2(b) and 2(c) (for instance, at temperatures around $180^{\circ} \mathrm{C}$ for compounds of Fig. 2). In fact, we used a thermoplastic poly-
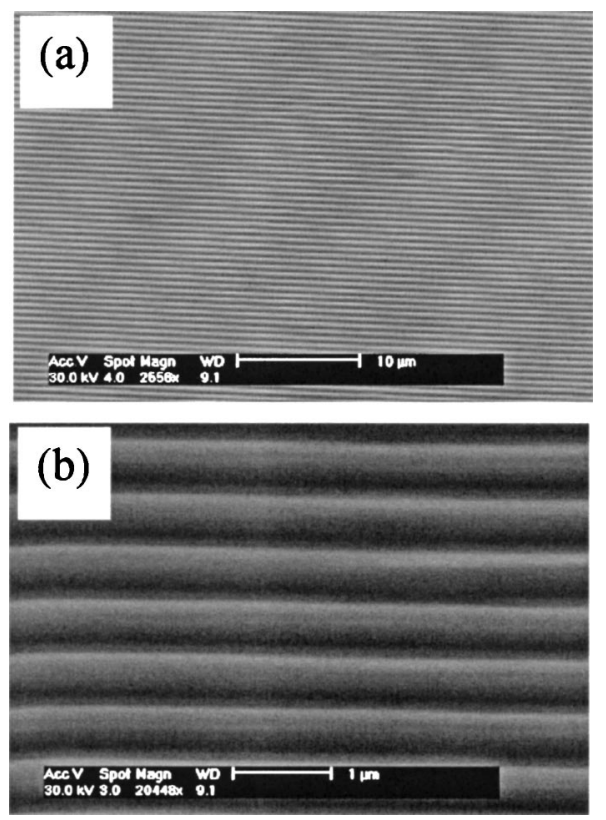

FIG. 3. SEM images of the pattern replicated into a high- $T_{g}$ polycarbonate element.
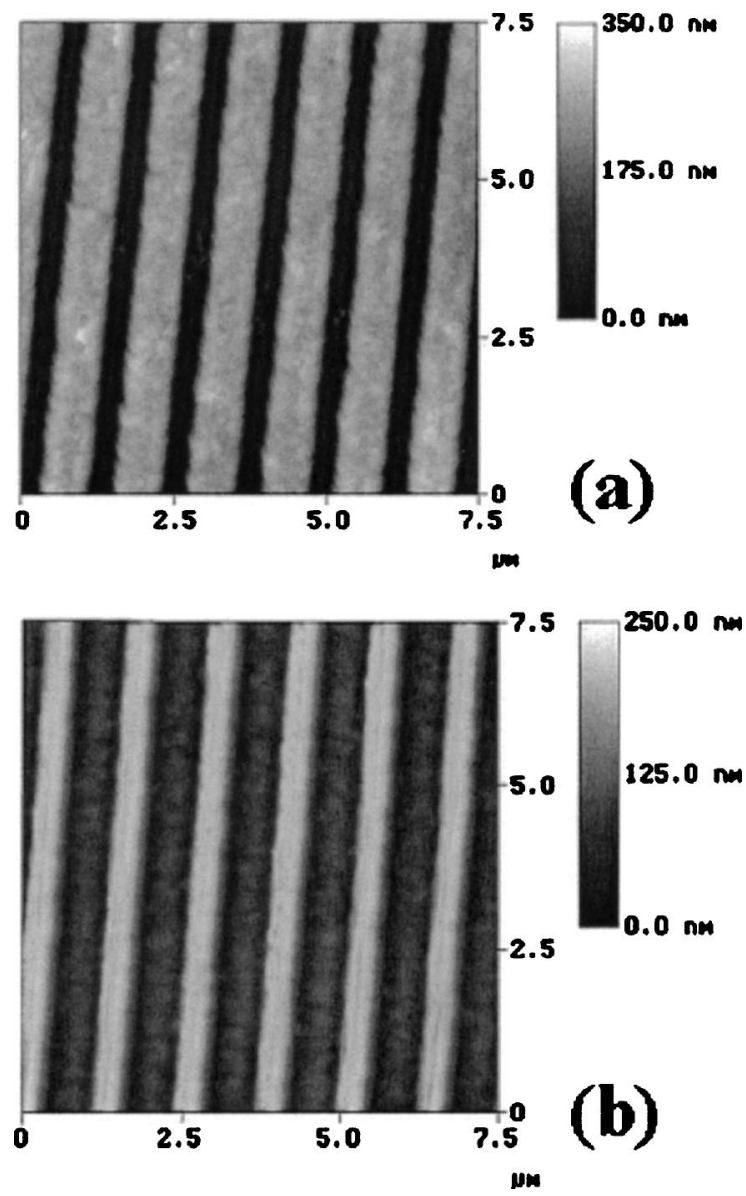

FIG. 4. Two-dimensional view of $1.2 \mu \mathrm{m}$ gratings imaged by AFM: (a) High- $T_{g}$ polycarbonate resin replica of the master pattern and (b) nanoimprinted PMMA $950 \mathrm{~K}$ gratings realized by using the high- $T_{g}$ polymeric molds.

carbonate resin with high glass transition temperature to subsequently imprint PMMA, which has $T_{g}$ between 120 and $130^{\circ} \mathrm{C}$.

In our experiments, polycarbonate in the glassy state was directly placed on the master and then the temperature was driven above the glass transition of the resin to allow a thermoplastic adhesion to the master, and consequently the pattern transfer to the replica. The uniformity over a large area of the replicated structure and its excellent definition are shown in Figs. 3(a) and 3(b), respectively. The final result of the replica molding process was a negative copy of the original pattern.

\section{Imprint procedure and results}

Figures 4(a) and 4(b) show the polymeric replica of the starting master and the final grating obtained on PMMA 950 $\mathrm{K}$, respectively, imaged by AFM. Comparing the image of the final grating (Fig. 5) with those of the quartz master (Fig. 5) demonstrates the good fidelity achieved with the replication procedure. The cross sections of the master, of the polycarbonate elements, and of the final polymer grating (Fig. 6), also show a good pattern transfer. $300 \mathrm{~nm}$ sized features 


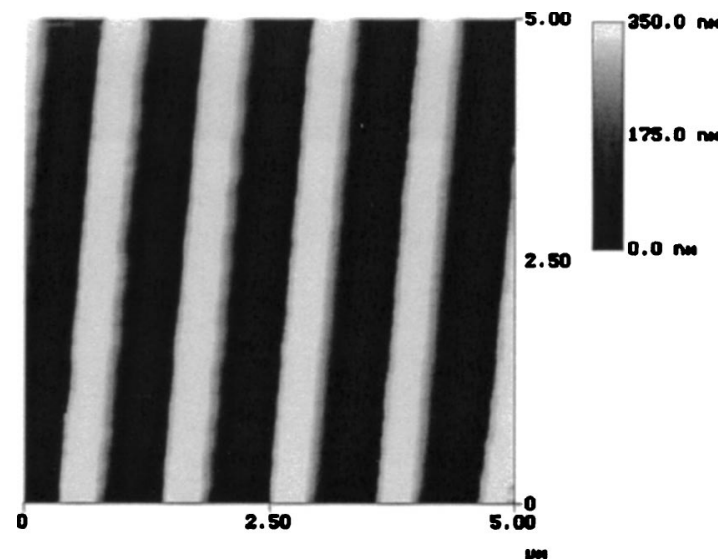

FIG. 5. AFM two-dimensional view of $1.2 \mu \mathrm{m}$ period quartz master grating.

were imprinted with the same fidelity onto areas up to several $\mathrm{cm}^{2}$. Similar results were obtained on PMMA $120 \mathrm{~K}$.

The observed variations in the periodicity of the master, the pressing mold, and the imprinted PMMA throughout the patterned areas are of less than 5\% (Figs. 4 and 6), which is comparable to the differences already present in the original masters. We observed that the deepness of the imprinted grooves can decrease with respect to that of the master
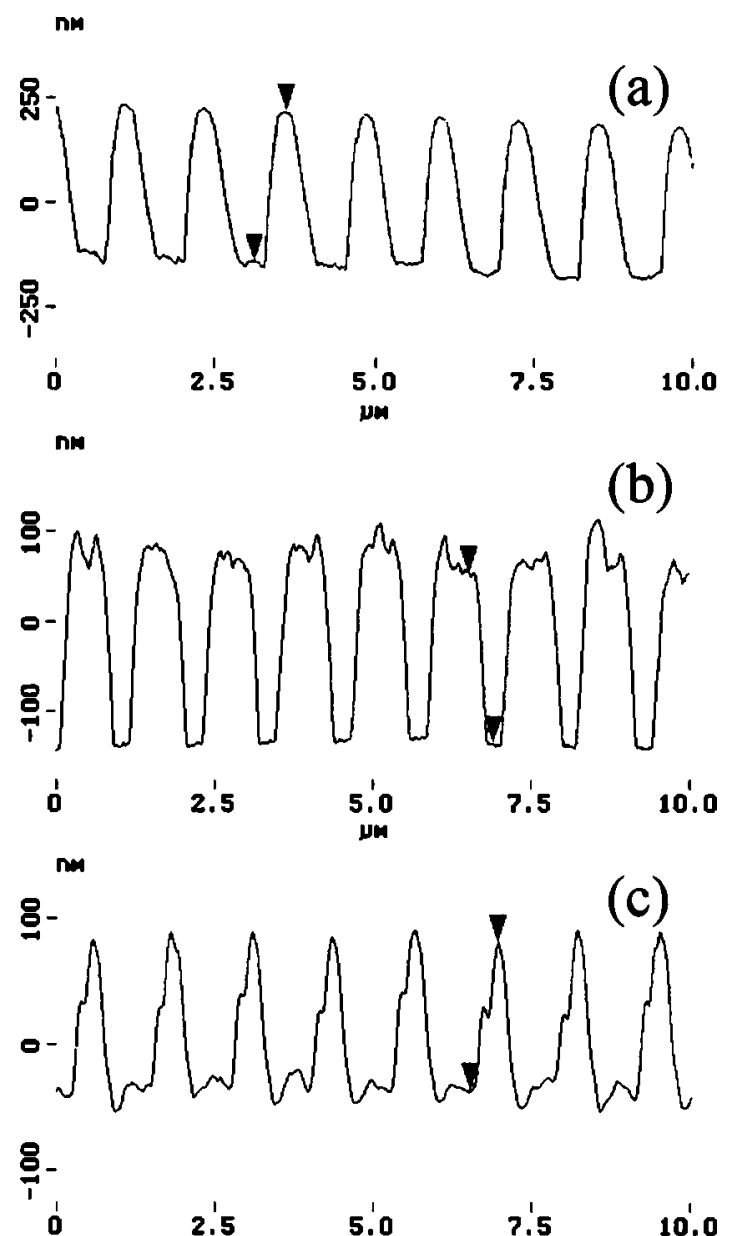

-

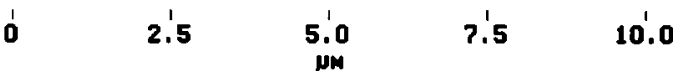

Fig. 6. AFM cross sections of the $1.2 \mu \mathrm{m}$ gratings: (a) Master, (b) polycarbonate replica, and (c) nanoimprinted PMMA $950 \mathrm{~K}$. (about $360 \mathrm{~nm}$ in Fig. 6), down to 190 and $120 \mathrm{~nm}$ for the polycarbonate replica and the PMMA grating, respectively (Fig. 6). Deeper features can be obtained by using longer heating times, both during the replica molding and during the final imprinting, which provides ample time to the polymers to move and to conform with the mold.

Moreover, we observe that, due to the incomplete filling of the recessed regions of the replica by the target polymer, double-crest features have been obtained on PMMA [Fig. 6(c)]. This is a general behavior of the imprinted polymers, which initially pile up in the regions adjacent to the protruding areas of the mold, and it can be observed in all different imprinting lithographies, including high-temperature, ${ }^{6}$ and room-temperature ${ }^{16}$ nanoimprinting, and soft molding. ${ }^{4}$ The cross-sectional profile of Fig. 6(c) shows that the two crests of the imprinted features have quite different heights, likely because of a slight slant of the pressing setup. The incomplete filling is also the reason for the roughness observed on the top of the features of the replicated mold and, consequently, on the bottom of the final target polymer. The rootmean-square roughness was found to slightly increase from $11 \mathrm{~nm}$ (bottom of the features of the master) to $14 \mathrm{~nm}$ (top of the mold and bottom of the imprinted PMMA). Finally, comparing Figs. 6(a) and 6(b), one notes a slight change in the shape of the features, which have rounded tops in the master and flatter bottoms in the replica. We believe that these differences are due to the fact that no pressure is applied on the system during the mold replication on polycarbonate, which can affect the fidelity of the pattern transfer. Also, these effects can be reduced by longer thermal cycles.

Unlike conventional NIL, which produces a negative copy of the master features on the target polymer, this approach uses intermediate replicas to transfer the pattern thus preserving the same shape (positive) of the master on the target polymer. Moreover, since the pressing mold for nanoimprinting is replicated instead of being fabricated, this allows for a faster mold fabrication and transfer procedure, whose overall duration is of a few minutes, similarly to soft lithographies employing elastomeric replicas. We could not find any significant distortion of the polymer mold upon imprinting, and the polycarbonate elements were used for many imprinting cycles (up to five) without appreciable differences in the final pattern transfer. The replica molding also prolongs the life of the original master, avoiding the stresses due to the direct use in the imprinting step. Further study is in progress in our laboratory to assess the general validity of this technique, and its applicability to different target polymers.

\section{SUMMARY AND CONCLUSION}

In this article, we report on a straightforward replica molding procedure exploiting the glass transition of high- $T_{g}$ polymers, which allows the replication of master patterns without using ultraviolet radiation, external pressure, and antisticking layers during the replica fabrication. The molding is carried out by driving the polymer above $T_{g}$, like an usual imprinting procedure, and the obtained rigid polymeric replicas are used to transfer pattern into PMMA by NIL. The 
process can be applied to any polymer having $T_{g}$ considerably lower than the softness temperature of the replicated master, with high fidelity of the pattern transfer. This approach to fabricating polymeric replicas allows a longer life of the master fabricated by photo- or electron-beam lithography, thus offering lower cost, higher throughput, and fast operation in comparison with other imprint lithography methods.

\section{ACKNOWLEDGMENTS}

This work has been partially funded by Agilent Technologies. The authors would like to thank G. De Stefanis and S. Bernardi for helpful discussion and A. Zocco and A. Perrone for the SEM investigation.

${ }^{1}$ S. Y. Chou, P. R. Krauss, and P. J. Renstrom, Appl. Phys. Lett. 67, 3114 (1995).

${ }^{2}$ S. Y. Chou, P. R. Krauss, and P. J. Renstrom, Science 272, 85 (1996).

${ }^{3}$ A. Kumar and G. M. Whitesides, Appl. Phys. Lett. 63, 2002 (1993).

${ }^{4}$ L. Stolz Roman, O. Inganäs, T. Granlund, T. Nyberg, M. Svensson, M. R. Andersson, and J. C. Hummelen, Adv. Mater. (Weinheim, Ger.) 12, 189 (2000); K. Y. Suh, Y. S. Kim, and H. H. Lee, ibid. 13, 1386 (2001).
${ }^{5}$ J. A. Rogers, M. Meier, A. Dodabalapur, E. J. Laskowski, and M. A. Cappuzzo, Appl. Phys. Lett. 74, 3257 (1999).

${ }^{6} \mathrm{~S}$. Zankovych, T. Hoffmann, J. Seekamp, J.-U. Bruch, and C. M. Sotomayor Torres, Nanotechnology 12, 91 (2001).

${ }^{7}$ M. Li, L. Chen, and S. Y. Chou, Appl. Phys. Lett. 78, 3322 (2001).

${ }^{8}$ L. Guo, P. R. Krauss, and S. Y. Chou, Appl. Phys. Lett. 71, 1881 (1997).

${ }^{9}$ Z. Yu, S. J. Schablitsky, and S. Y. Chou, Appl. Phys. Lett. 74, 2381 (1999).

${ }^{10}$ S. Y. Chou, P. R. Krauss, W. Zhang, L. Guo, and L. Zhuang, J. Vac. Sci. Technol. 15, 2897 (1997).

${ }^{11}$ Y. Xia and G. M. Whitesides, Angew. Chem., Int. Ed. Engl. 37, 550 (1998).

${ }^{12}$ H. Schulz, D. Lyebyedyev, H.-C. Scheer, K. Pfeiffer, G. Bleidiessel, G. Grutzner, and J. Ahopelto, J. Vac. Sci. Technol. B 18, 3582 (2000).

${ }^{13} \mathrm{We}$ observed in PMMA $950 \mathrm{~K}$ tendency to adhere to the polymer mold, notwithstanding the OTS treatment, for temperatures higher than $T_{g}$. For this reason, the NIL process on PMMA $950 \mathrm{~K}$ was performed at temperatures slightly below $T_{g}\left(\cong 100{ }^{\circ} \mathrm{C}\right)$, with a successful pattern transfer [Fig. $5(\mathrm{~b})]$.

${ }^{14}$ H. Vogel, Phys. Z. 22, 645 (1921); G. S. Fulcher, J. Am. Ceram. Soc. 8, 339 (1925); G. Tamman and W. Hesse, Z. Anorg. Allg. Chem. 156, 245 (1926).

${ }^{15}$ K. L. Ngai and D. J. Plazek, Physical Properties of Polymers Handbook, edited by J. E. Mark (American Institute of Physics, Melville, 1996).

${ }^{16}$ P. S. Hong and H. H. Lee, Appl. Phys. Lett. 83, 2441 (2003). 